\title{
Hypoxic Pretreatment of Adipose-Derived Stem Cells Accelerates Diabetic Wound Healing via circ-Gcap14 and HIF-1 $\alpha$ /VEGF Mediated Angiopoiesis
}

\author{
Zhi Wang, Cheng Feng, Hao Liu, Tian Meng, Weiqing Huang, Xiao Long, Xiaojun Wang \\ Department of Plastic E Cosmetic Surgery, Peking Union Medical College Hospital, Beijing, China
}

\begin{abstract}
Background and Objectives: Adipose-derived stem cell (ADSC) transplantation improves stem cell paracrine function and can enhance wound healing. However, in diabetic patients, glucose-associated effects on this function and cell survival lead to impaired wound closure, thereby limiting ADSC transplantation efficiency. The hypoxia-inducible factor HIF-1 $\alpha$ has an important protective function during wound healing. Here, we aim to clarify the regulatory mechanism of ADSCs.

Methods and Results: ADSCs were isolated from BALB/C mice adipose samples. We then used high-throughput sequencing to assess abnormal expression of circular RNAs (circRNAs). We also used an in vivo full-thickness skin defect mouse model to assess the effects of transplanted ADSC on diabetic wound closure. Hypoxic pretreatment of ADSCs accelerated diabetic wound closure, which enhanced angiogenic growth factor expression in our mouse model. High-throughput sequencing and RT-qPCR indicated that circ-Gcap14 was upregulated in hypoxic pretreated ADSCs. Similarly, circ-Gcap14 downregulation also decreased the therapeutic effects of ADSCs; however, circ-Gcap14 overexpression increased the effects of ADSC by promoting angiopoiesis. We also used a luciferase reporter assay to confirm that miR-18a-5p and HIF-1 $\alpha$ were downstream targets of circ-Gcap14. HIF-1 $\alpha$ expression plays an important role in increased VEGF level.

Conclusions: Based on our data, we suggest that circ-Gcap14 plays an important role in accelerating hypoxic ADSC-mediated diabetic wound closure, by enhancing mouse angiogenic growth factor expression and regulating downstream miR-18a-5p/HIF-1 $\alpha$ expression.
\end{abstract}

Keywords: circ-Gcap14, HIF-1 $\alpha$, Angiopoiesis, Diabetic wound healing, ADSCs

Received: March 13, 2021, Revised: June 23, 2021, Accepted: July 1, 2021, Published online: August 31, 2021

Correspondence to Zhi Wang

Department of Plastic \& Cosmetic Surgery, Peking Union Medical College Hospital, No.1 Shuaifuyuan, Dongcheng District, Beijing 100730, China

Tel: +86-10-69158730, Fax: +86-10-69158732, E-mail: wangzh@pumch.cn

Co-Correspondence to Xiaojun Wang

Department of Plastic \& Cosmetic Surgery, Peking Union Medical College Hospital, No.1 Shuaifuyuan, Dongcheng District, Beijing 100730 China

Tel: +86-10-69158730, Fax: +86-10-69158732, E-mail: pumchwxj@163.com

다 This is an open-access article distributed under the terms of the Creative Commons Attribution Non-Commercial License (http://creativecommons.org/licenses/by-nc/4.0/), which permits unrestricted non-commercial use, distribution, and reproduction in any medium, provided the original work is properly cited.

Copyright (c) 2021 by the Korean Society for Stem Cell Research 


\section{Introduction}

Diabetes mellitus (DM) is a severe chronic metabolic disease reflected by high glucose levels that induce oxidative stress. Globally, DM affects $>400$ million people, and cases are expected to increase by $50 \%$ by 2030 (1-3). Micro- and macrovascular traits are the main diabetic complications and are the key mortality risk factors for diabetic patients (4). Previous research has shown that adipose-derived stem cell (ADSC) transplantation therapy promotes diabetic wound closure (5). However, high glucose microenvironments in diabetic patients inhibit ADSC survival, resulting in poor treatment outcomes (6). Therefore, efficient mechanisms that enhance stem cell survival in these inclement microenvironments must be delineated.

Increasingly, studies have observed that hypoxia pretreatment promotes ADSC proliferation via increased vascular endothelial growth factor (VEGF) levels (7); however, the regulatory mechanisms for this effect remain unclear. A recent study observed that circular RNAs (circRNAs) actively participate in pathological mechanisms across different disorders. CircRNAs are more stable than linear RNAs and are highly expressed in some tissues; thus, they are considered a more promising potential biomarker than linear RNAs (8). Previously, circRNAs were reported to function as miRNA 'sponges' that naturally and competitively sequester and suppress miRNA activity (9-11). However, how circRNAs function in ADSC-mediated diabetic wound healing remains unclear. Therefore, we employed high-throughput sequencing, RT-qPCR and a diabetic wound mouse model to study circRNAs in ADSC acceleration of diabetic wound healing.

\section{Materials and Methods}

\section{Ethics statement}

The Animal Care and Use Committee of Peking Union Medical College Hospital Hospital approved the investigation protocol (XHDW-2020-01). We performed all postoperative animal care and surgical interventions according to the NIH guide for Care and Use of Laboratory Animals.

\section{ADSC isolation and identification}

ADSCs were isolated from mouse adipose tissue as previously described $(12,13)$. We observed no uninduced differentiation during our cell culture expansion. We induced osteogenic differentiation in 3-week ADSC cultures in Dulbecco's modified Eagle's medium (DMEM) (Invitrogen, Carlsbad, CA, USA), supplemented with $10 \%$ fetal bovine serum (FBS) (Invitrogen, Carlsbad, CA, USA), $0.1 \mu \mathrm{M}$ dexamethasone, $50 \mu \mathrm{M}$ ascorbate-2-phosphate and $10 \mathrm{mM} \beta$-glycerophosphate. We induced adipogenic differentiation by culturing ADSCs for two weeks in DMEM supplemented with $10 \% \mathrm{FBS}, 10 \mu \mathrm{M}$ insulin, $0.5 \mathrm{mM}$ isobutylmethylxanthine, $200 \mu \mathrm{M}$ indomethacin and $1 \mu \mathrm{M}$ dexamethasone. ADSC osteogenic or adipogenic differentiation was investigated using alizarin red (Invitrogen, Carlsbad, CA, USA) and oil-red O (Invitrogen, Carlsbad, CA, USA) staining. We grew normoxic ADSCs cultures in $95 \%$ air $\left(20 \% \mathrm{O}_{2}\right)$ and $5 \% \mathrm{CO}_{2}$. For hypoxic pretreatment, ADSCs were cultures in $93 \% \mathrm{~N}_{2}, 2 \% \mathrm{O}_{2}$ and $5 \% \mathrm{CO}_{2}$.

\section{Strand-specific and high-throughput RNA-Seq library construction}

Total RNA from ADSCs and hypoxic-pretreated ADSCs was isolated using TRIzol reagent (Invitrogen, Carlsbad, CA, USA). Approximately $3 \mu \mathrm{g}$ total RNA per sample was prepared using a VAHTS Total RNA-seq (H/M/R) Library Prep Kit from Illumina (Vazyme Biotech Co., Ltd, Nanjing, China). This kit isolated ribosomal RNA and removed the remaining RNAs, including non-coding RNA and mRNA. We then performed an RNA purification step using RNase R (Epicenter, $40 \mathrm{U}$ at $37^{\circ} \mathrm{C}$ for $3 \mathrm{~h}$ ), followed by TRIzol. An RNA-seq library was established using a KAPA Strand RNA-Seq Library Prep Kit (Roche, Basel, Switzerland) and expose them in order for extensive codifying with Illumina HiSeq 4000 from Aksomics, Inc. (Shanghai, China).

\section{Diabetic wound induction}

We used $\mathrm{BALB} / \mathrm{C}$ mice to induce a diabetic state via a single intraperitoneal injection of $60 \mathrm{mg} / \mathrm{kg}$ streptozotocin (STZ, Sigma, USA) dissolved in $0.1 \mathrm{M}$ citrate buffer (pH 4.5). On day 7 after STZ administration, we validated the presence of diabetes by assessing fasting blood glucose levels in blood samples from tail veins. A mouse with a fasting blood glucose level of $>250 \mathrm{mg} / \mathrm{dl}$ was considered diabetic. Animals were maintained for one month and were used for subsequent analyses of posterior blood glucose stabilization. Following diabetes confirmation, we anesthetized mice by intramuscular injection of $30 \mathrm{mg} / \mathrm{kg}$ sodium pentobarbital. Once anesthetized, the hair was shaved from the dorsal leg area and the region sterilized using povidone iodine solution. A sterile biopsy punch was used to generate a 4-mm full-thickness excisional wound. After this procedure, we randomly allocated mice 
to subcutaneous injection with $100 \mu 1$ PBS containing $1 \times 10^{5} \mathrm{ADSCs}$, or an equal volume of PBS at four sites near the wound $(25 \mu \mathrm{l} / \mathrm{site})$. We euthanized mice after three weeks and harvested skin samples for histopathological analyses.

\section{CD31 immunohistochemistry}

We fixed, processed, and blocked skin sections for immunofluorescence staining. Sections were incubated overnight at $4^{\circ} \mathrm{C}$ with an anti-rabbit CD31 antibody, followed by incubation with biotinlyated goat anti-mice IgG antibody (Vector laboratories). Following secondary antibody incubation, we incubated sections in avidin:biotinylated enzyme complex (ABC; Vector Laboratories) for $30 \mathrm{~min}$. We then treated sections with 3,3'-diaminobenzidine substrate (Vector Laboratories), and the section produced a brown stain. We mounted slides using Fluka Eukitt quick-hardening mounting medium (Sigma Aldrich). Negative controls contained no primary antibodies. Images were captured using a Carl Zeiss MIRAX MIDI via a Plan-Apochromat $20 \times / 0.8 \mathrm{NA}$ objective lens and Marlin F146.C camera, and images were processed in MIRAX Viewer Version 1.11.49.0 software (Carl Zeiss Microimaging $\mathrm{GmbH}$, Oberkochen, Germany).

\section{RNA overexpression or interference}

We induced miR-18a-5p overexpression using miR-18a-5p mimics. We purchased a circ-Astnl overexpression vector and HIF-1 $\alpha$ silencing vector from RiboBio (Guangzhou, China). We performed all cell transfections using Lipofectamine 2000 (Thermo Fisher Scientific), following a previous method (14).

\section{Western blot assay}

Skin tissues were lysed, and lysates were centrifuged at $12,000 \mathrm{rpm}$ at $4^{\circ} \mathrm{C}$ following addition of a protease inhibitor. The protein concentration was determined with a Pierce bicinchoninic acid assay (BCA) kit (Thermo Fisher). Proteins was separated by $10 \%$ SDS-PAGE and transferred to PVDF membranes. The primary antibodies used to assay protein expression were VEGF (1:6S00), HIF-1 $\alpha$ (1: 600) (all Santa Cruz Biotechnology, Dallas, TX, USA), Anti-GAPDH (1 : 1,000, Sigma-Aldrich). Horseradish peroxidase-conjugated secondary antibody $(1: 1,000$, Abcam, USA). An ECL chemiluminescent kit (Millipore, Burlington, MA, USA) was used to read the bands.

\section{Quantitative real-time polymerase chain reaction (RT-qPCR)}

Total RNA was isolated from wound tissues and cells using a TRIzol reagent kit. We synthesized and amplified cDNA using a TaqMan miRNA Reverse Transcription Kit. RT-qPCR reactions were performed using a TaqMan Human miRNA Assay Kit, using the $2^{-\Delta \Delta \mathrm{CT}}$ method to detect expression fold changes. We used U6 and GAPDH genes as internal references. Primers were: circ-Gcap14, F: 5'-GCAGCTATGAGTCCTCTG-3', R, 5'-CTTCCATGGGC TATAAGGTG-3'; HIF-1 $\alpha$, F: 5'-AGAGTCAAGCCCAGAG TCAC-3', R, 5'-TGGGACTGTTAGGCTCAGGT-3'; VEGF, F: 5'-GCACCCATGGCAGAAGGAGGAG-3', R, 5'-GTGC TGACGC-TAACTGACC-3'; miR-18a-5p, F: 5'-CAGTAAA GGTAAGGAGAGCTCAATCTG-3', R: 5'-CATACAACCA CTAAGCTAAAGAATAATCTGA-3; U6, F: 5'-AGTAAGC CCTTGCTGTCAGTG-3', R: 5'-CCTGGGTCTGATAATG CTGGG-3'; GAPDH: F: 5'-GTCTCCTCTGACTTCAACA GCG-3', R: 5'-ACCACCCTGTTGCTGTAGCCAA-3' (Gene Pharma, Shanghai, China).

\section{Luciferase reporter assay}

We cloned wild-type (wt) and 3'-UTR mutant (mut) HIF-1 $\alpha$, and wt and mut circ-Gcapl4 into pMIR firefly luciferase-expressing vectors. We co-transfected vectors into $70 \%$ confluence HEK293T cells. We used $500 \mathrm{ng}$ pMIR-HIF-1 $\alpha$-wt/pMIR-HIF-1 $\alpha$-Mut or pMIR-circ-Gcap14$\mathrm{wt} / \mathrm{pMIR}$-circ-Gcap14-mut combined with $50 \mathrm{nM}$ miR-18a$5 \mathrm{p}$ mimics. We assayed luciferase activity using the Dual-Luciferase Reporter System (Promega, Madison, WI, USA) and performed five independent assays.

\section{Statistical analyses}

We expressed continuous parameters using the mean \pm standard deviation (SD), and used one-way variance analysis (ANOVA) to compare data in GraphPad Prism 5.1. A $\mathrm{p}$ value $\leq 0.05$ indicated statistical significance.

\section{Results}

The hypoxic pretreatment increases the therapeutic effect of ADSCs to accelerates diabetic wound closure

ADSCs can effectively repair diabetic wounds (15). We observed that ADSCs possessed classical cobblestone-like morphology (Fig. 1A). Immunofluorescence staining was positive for the mesenchymal cell markers CD44, CD90, CD29 and CD105, and negative for the endothelial markers CD31 and von Willebrand Factor (vWF) (Fig. 1B G). Alizarin red and oil red $\mathrm{O}$ staining also confirmed that ADSCs exhibited osteoblast and adipocyte differentiation abilities (Fig. 1H and 1I).

A previous study indicated that the hypoxic pretreatment of ADSCs improved therapeutic efficacy (7). To clar- 
A

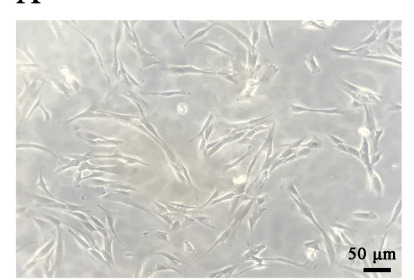

D

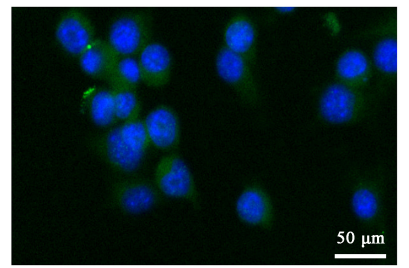

CD90

G

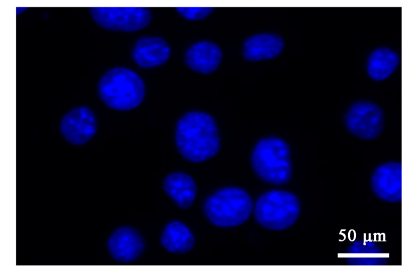

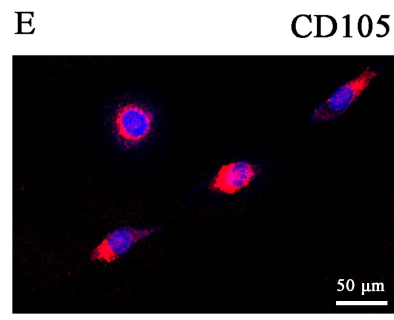

B

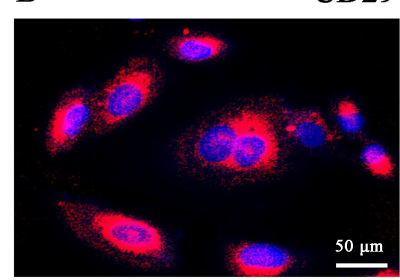

WF H

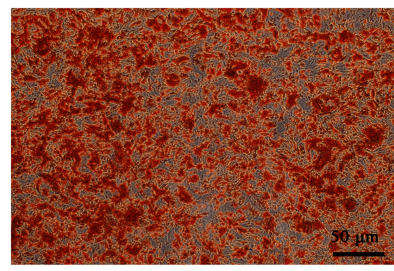

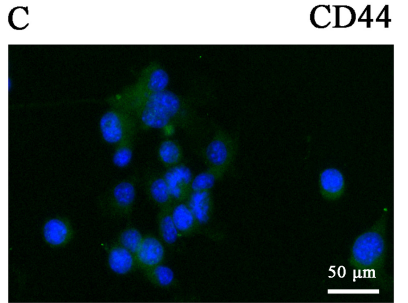

$\mathrm{F}$

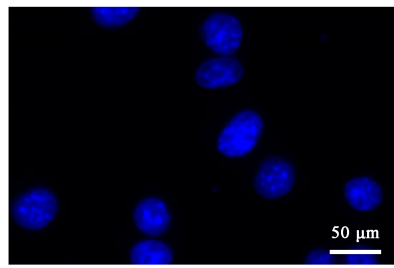

I

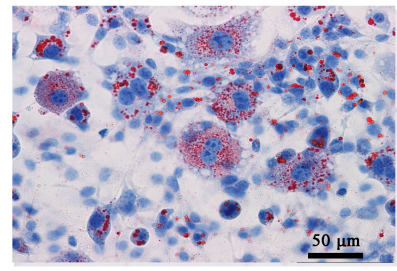

Fig. 1. Characterization of adiposederived mesenchymal stem cells (ADSCs). (A) ADSCs show a typical cobblestone-like morphology. $(\mathrm{B} \sim \mathrm{G})$ Immunofluorescence staining of cell surface markers. Antibodies were labeled with either fluorescein isothiocyanate (FITC, green) or phycoerythrin (PE, red). CD29, CD44, CD90, and CD105 staining was positive, whereas CD31 and von Willebrand Factor (vWF) staining was negative. $(\mathrm{H}, \mathrm{I})$ Differentiation potential of ADSCs by oil red $\mathrm{O}(\mathrm{H})$ and alizarin red staining (I). Scale bar, $50 \mu \mathrm{m}$. ify if hypoxic pretreatment of ADSCs could indeed accelerate diabetic wound closure, mice were injected with STZ for one week to construct a diabetic mouse model. We anesthetized mice with sodium pentobarbital $(0.5 \mathrm{mg} / \mathrm{g})$, and made a $10 \mathrm{~mm}$ full-thickness round excisional skin wound on every mouse instep, with a sterile $10-\mathrm{mm}$ punch biopsy tool. Following received control ADSCs or hypoxia-pretreated ADSCs, diabetic wound closure was detected 0, 7 and 14 day after surgery (Fig. 2A). Our results showed that hypoxic pretreatment of ADSCs accelerated diabetic wound closure (Fig. 2B and 2C). Immunohistochemical CD31 staining showed that this pretreatment promoted angiogenesis (Fig. 2D and 2E). RT-qPCR and western blot analyses showed that hypoxic pretreatment of ADSCs significantly increased HIF-1 $\alpha$ and VEGF expression (Fig. $2 \mathrm{~F}$ and $2 \mathrm{G}$ ). These data suggested that hypoxic ADSC pretreatment accelerated diabetic wound closure, and increased angiogenic growth factor expression in our mouse model.

\section{Circ-Gcap14 played an important role in hypoxic-ADSC accelerated diabetic wound closure, and enhanced angiogenic expression in our mouse model}

CircRNAs have important functions in microenvironmental regulation (16). In our study, high-throughput sequencing was used to explore if circRNAs play a role in
ADSC-accelerated diabetic wound closure. Our data revealed that ADSC hypoxic pretreatment resulted in several differentially expressed circRNAs (Fig. 3A). RT-qPCR analysis showed that circ-Gcap14 expression was significantly increased in hypoxic-ADSCs when compared with non-hypoxic cells (Fig. 3B).

We then constructed a circ-Gcap14 overexpression and silencing vector, and transfected both into ADSCs. As expected, our data showed that circ-Gcap14 expression was significantly increased after transfection with the circGcap14 overexpression vector, but was significantly silenced upon circ-Gcap14 downregulation (Fig. 4A). After ADSC pretreatment with hypoxia, circ-Gcap14 overexpression significantly accelerated diabetic wound closure, but circ-Gcap14 silencing decreased diabetic wound closure (Fig. 4B and 4C). Immunohistochemical staining for CD31 showed that circ-Gcap14 overexpression promoted angiogenesis, but circ-Gcap14 silencing decreased it (Fig. 4D and 4E). RT-qPCR and western blot analysis also showed that circ-Gcap14 overexpression increased VEGF and HIF-1 $\alpha$ expression, while circ-Gcap14 silencing decreased this expression (Fig. 4F and 4G). These data suggested that circ-Gcap14 functions in hypoxic-ADSC acceleration of diabetic wound closure, and enhances angiogenic growth factor expression in our mouse model. 
A

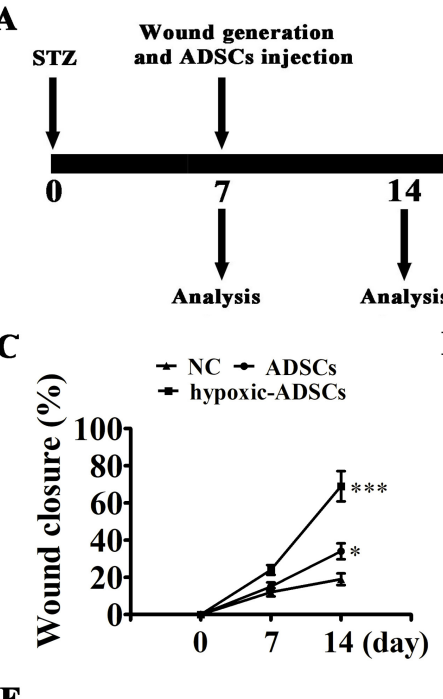

$\mathbf{F}$

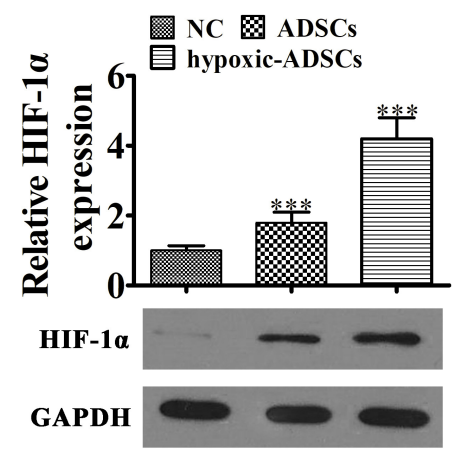

B

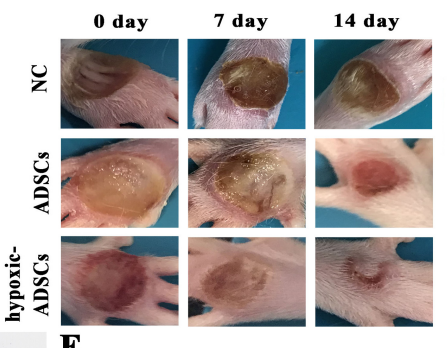

D
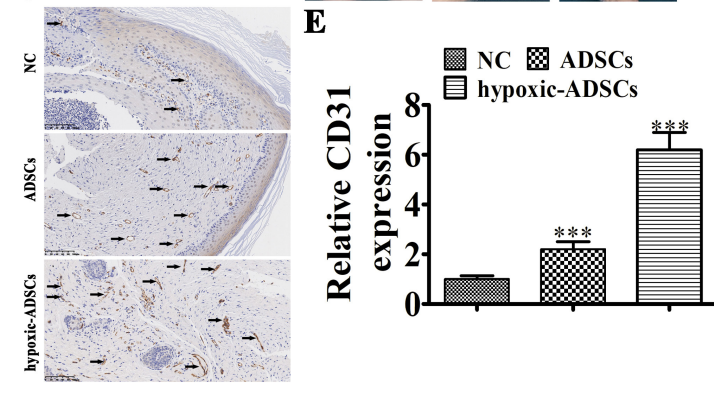

$\mathbf{G}$

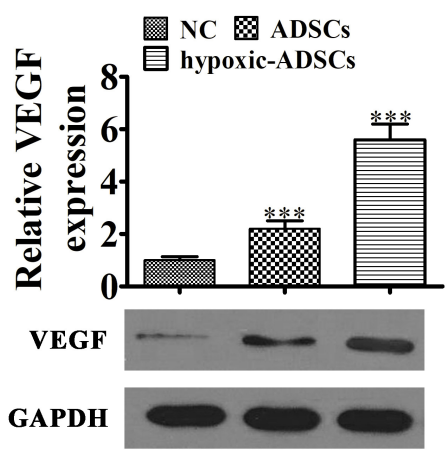

A

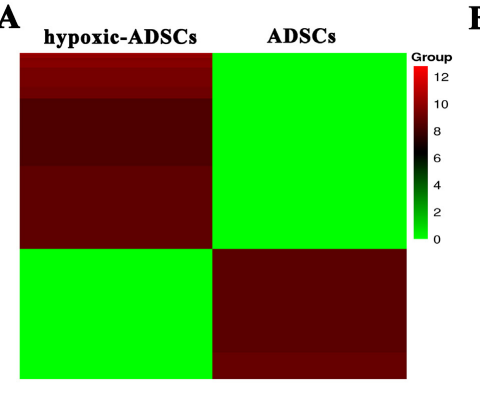

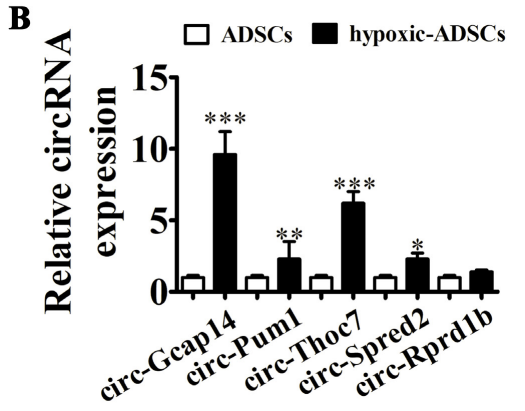

Fig. 2. Wound healing effects of hypoxia-pretreated ADSCs in mice. (A) Schematic of in vivo procedures. (B, C) Wound closure rates were quantified at indicated times after wound generation and ADSC transplantation. Data are presented as the mean $\pm \mathrm{SD}$. ${ }^{*} \mathrm{p}<0.05, * * * \mathrm{p}<0.001$ vs. NC group. (D, E) CD31 immunohistochemical staining detection the angiogenesis as black arrow show. Data are presented as mean \pm SD. ${ }^{* * *} \mathrm{p}<0.001$ vs. NC group. $(F, G)$ RT-qPCR and western blot detection show the expression HIF- $1 \alpha$ and VEGF in mRNA and protein level from tissue surrounding wounds in the diabetic mouse model. Data are presented as the mean \pm SD. ${ }^{* * *} p<0.001$ vs. NC group.

Fig. 3. Circ-Gcap14 is upregulated in hypoxia-pretreated ADSCs (hypoxicADSCs). (A) Heat map of upregulated and downregulated circRNAs with a $\geq 1$.5-fold difference between hypoxic- and wild-type ADSCs. (B) RTqPCR shows circRNA expression in both hypoxic- and wild-type ADSCs. Data are presented as the mean \pm SD. ${ }^{* *} \mathrm{p}<0.01,{ }^{* * *} \mathrm{p}<0.001$ vs. ADSCs group.
The expression of circ-Gcap14 increased HIF-1 $\alpha$ level by adsorption miR-18a-5p

Bioinformatics data showed that circ-Gcapl4 regulates HIF-1 $\alpha$ expression via miR-18a-5p inhibition. HIF-1 $\alpha$ is involved in angiogenesis by promoting VEGF expression $(17,18)$. To validate interactions between circ-Gcap14, miR-18a-5p and VEGF, we created a suite of luciferase reporter (LR) vectors. MiR-18a-5p binding sites on circGcap14, as well as sites with point mutations were added to prevent binding (Fig. 5A). By performing the luciferase assay in mut or wt circ-Gcap14 transfected HEK293T cells, we showed that miR-18a-5p suppressed circ-Gcap14 activity (Fig. 5B).

We next created the $L R$ vector. Candidate miR-18a-5p binding sites regarding HIF-1 $\alpha$ 3'-UTR and those with point mutations inserted to prevent binding were constructed (Fig. 5C). We transfected HEK293T cells with mut or wt HIF-1 $\alpha$ 3'-UTR and assayed luciferase activity, demonstrating that wt miR-18a-5p suppressed HIF-1 $\alpha$ activity (Fig. 5D). RT-qPCR analyses also showed that circ-Gcap14 overexpression promoted circ-Gcap14 and HIF-1 $\alpha$ expression, but decreased miR-18a-5p expression 
A

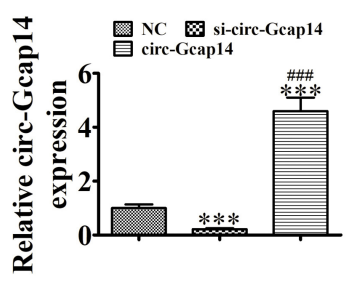

C

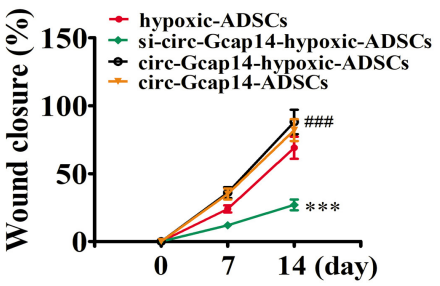

B

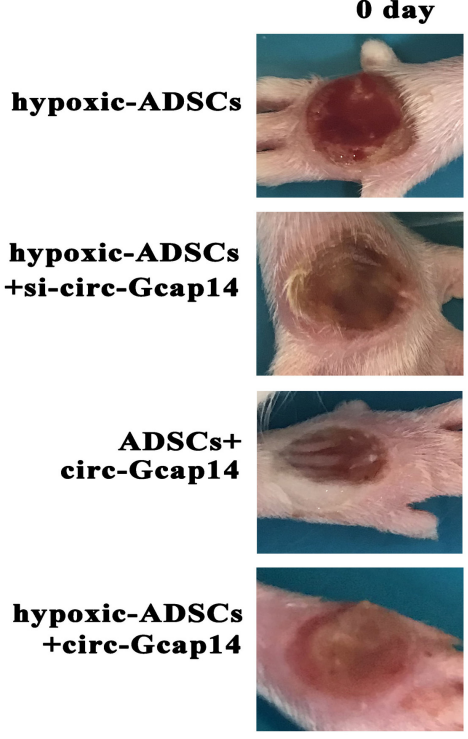

7 day
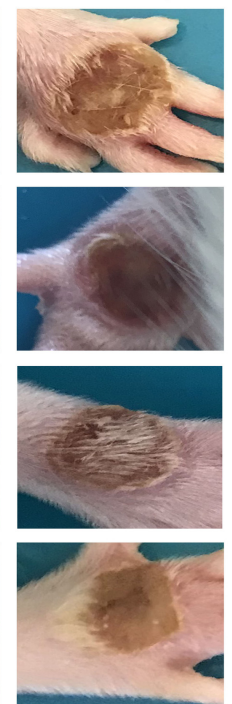

14 day
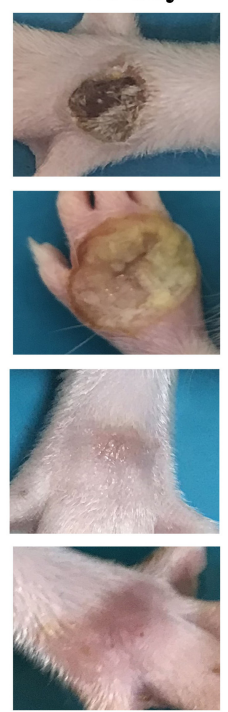

D

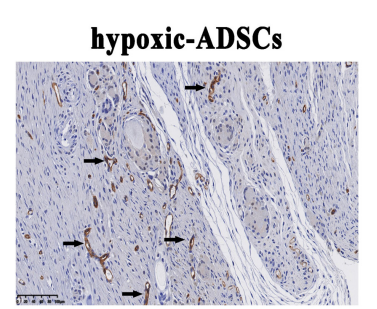

$\mathbf{E}$

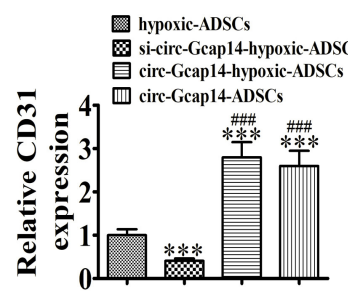

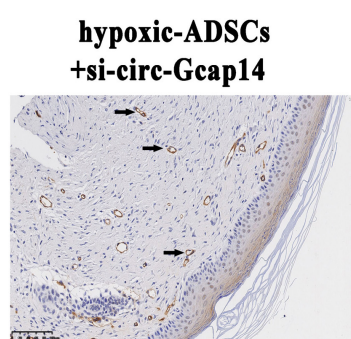

$\mathbf{F}$

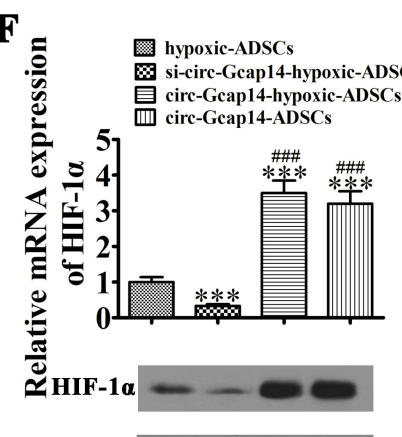

ADSCs +circ-Gcap14

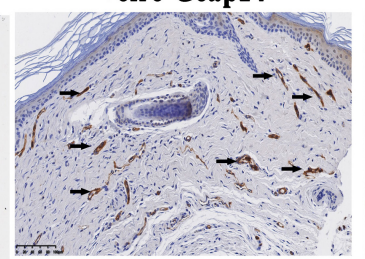

G

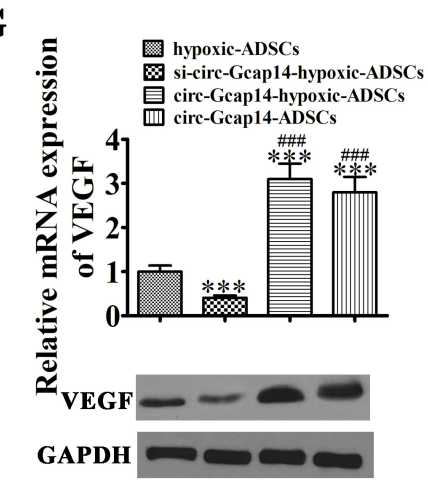

Fig. 4. Circ-Gcap14 plays an important role in ADSC mediated diabetic wound closure. (A) RT-qPCR shows circ-Gcap14 expression in ADSCs upon circ-Gcap14 downregulation or overexpression/silencing. Data are presented as the mean \pm SD. $* * * p<0.001$ vs. NC. ${ }^{\# \# \#} \mathrm{p}<0.001$ vs. si-circ-Gcap14. (B, C) The rate of wound closure was quantified at indicated times after ADSC transplantation. Data are presented as the mean \pm SD. ${ }^{* * *} \mathrm{p}<0.001$ vs. hypoxic-ADSC. ${ }^{\# \#} \mathrm{p}<0.001$ vs. si-circ-Gcap14-hypoxic-ADSC. (D, E) CD31 immunohistochemical staining detection the angiogenesis as black arrow show. Data are presented as the mean $\pm \mathrm{SD}$. $* * * p<0.001$ vs. hypoxic-ADSC. ${ }^{\# \#} \mathrm{p}<0.001$ vs. si-circ-Gcap14-hypoxic-ADSC. (F, G) RT-qPCR and western blot detection shows the expression of HIF- $1 \alpha$ and VEGF in both mRNA and protein level from tissue surrounding wounds. Data are presented as the mean \pm SD. $* * * p<$ 0.001 vs. hypoxic-ADSC. ${ }^{\# \# \#} p<0.001$ vs. si-circ-Gcap14-hypoxic-ADSC.

(Fig. 5E), suggesting that miR-18a-5p and HIF-1 $\alpha$ were downstream targets of circ-Gcap14. MiR-18a-5p upregulation suppressed HIF-1 $\alpha$ expression, but HIF-1 $\alpha$ overexpression did not reverse miR-18a-5p expression, suggesting that HIF-1 $\alpha$ was a miR-18a-5p downstream target
(Fig. 5F and 5G). These data showed that both miR-18a-5p and HIF-1 $\alpha$ were downstream circ-Gcapl4 targets. 
$\mathbf{A}$

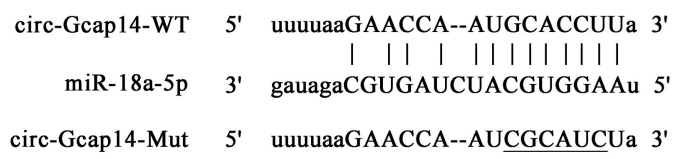

C

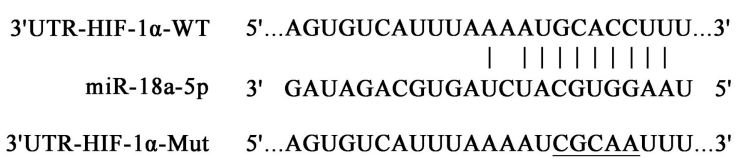

$\mathbf{E}$

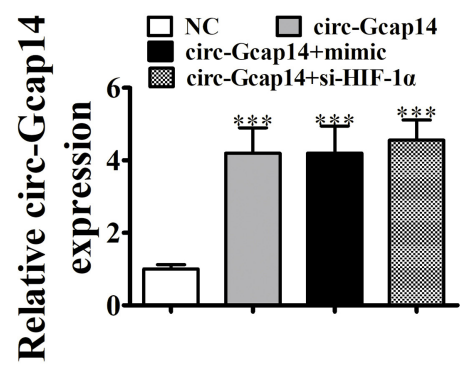

F

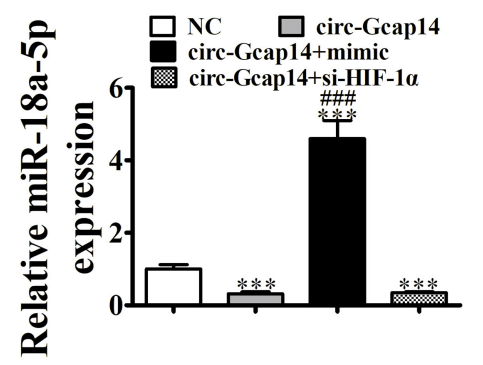

B

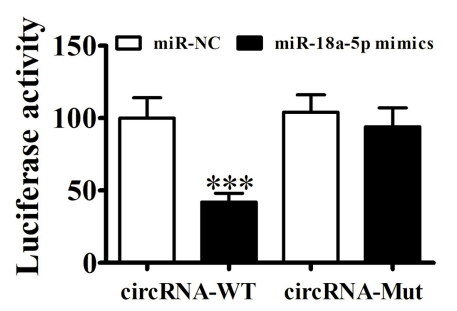

D

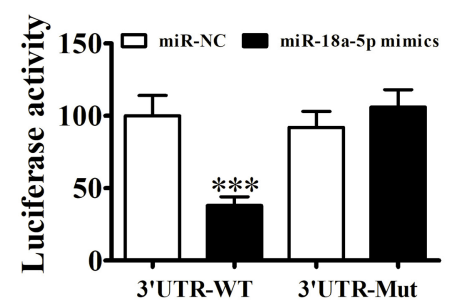

G

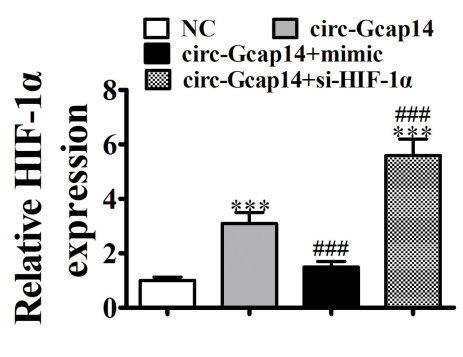

Fig. 5. miR-18a-5p and HIF-1 $\alpha$ are downstream targets of circ-Gcap14. (A) Predicted binding sites for miR-18a-5p in circ-Gcap14. The mutated circ-Gcap14 is also shown. (B) Relative luciferase activity at $48 \mathrm{~h}$ post-transfection of HEK293T cells with miR-18a-5p mimics/NC or circ-Gcap14 wild-type/mutant. Data are presented as the mean $\pm \mathrm{SD}$. ${ }^{* * *} \mathrm{p}<0.001$. (C) Binding site prediction of miR-18a-5p in the HIF-1 $\alpha$ 3'UTR. The mutant version of the $3^{\prime}-$ UTR-HIF-1 $\alpha$ is shown. (D) Relative luciferase activity at $48 \mathrm{~h}$ post-transfection of HEK293T cells with miR-18a-5p mimic/NC or $3^{\prime}$ UTR-HIF-1 $\alpha$ wild-type/mutant. Data are presented as the mean \pm SD. ${ }^{* * *} p<0.001$. (E $\left.\sim \mathrm{G}\right)$ RT-qPCR shows circ-Gcap14 (E), miR-18a-5p (F) and HIF-1 $\alpha$ expression (G). Data are presented as the mean \pm SD. $* * * p<0.001$ vs. NC group. ${ }^{\# \# ~} \mathrm{p}<0.001$ vs. circ-Gcap14.

\section{Discussion}

In DM patients, acute skin wounds resulting from trauma usually turn to chronic non-healing wounds (19). Several methods have been tried to accelerate the diabetic wound healing process, but optimized therapeutic strategies are lacking (20). Previous investigations have suggested that ADSC transplantation therapy accelerates diabetic wound closure $(13,15)$. Thus, in this study, we observed that hypoxic pretreatment of ADSCs exerted greater acceleration effects on diabetic wound closure than non-hypoxic ADSCs. Under hypoxic pretreatment conditions, high-throughput sequencing identified circ-Gcap14 expression as critical in the process; downregulated/silenced circ-Gcap14 decreased ADSC therapeutic effects, whereas circ-Gcap14 overexpression increased these therapeutic effects, suggesting that circ-Gcap14 plays a role in the ADSC acceleration of diabetic wound closure.

Bioinformatics and luciferase reporter analyses found that HIF-1 $\alpha$ and miR-18a-5p were circ-Gcap14 downstream targets. The overexpression of circ-Gcap14 promoted HIF-1 $\alpha$ and miR-18a-5p downregulation. Previous studies have shown that VEGF is the main HIF- $1 \alpha$ downstream effector, inducing cell proliferation, migration and tube formation in endothelial cells (21-23). The HIF-1 $\alpha$ NEGF signaling pathway is a critical target for angiogenic-related disease therapies, e.g. diabetic ulcers (24, 25). Our study also confirmed that circ-Gcapl4 has an important role in hypoxic-ADSC acceleration of diabetic wound closure, and enhances VEGF expression.

In conclusion, our study indicates that the hypoxic pretreatment of ADSCs enhances wound healing in a diabetic mouse model via induction of the circ-Gcapl4/miR-18a$5 \mathrm{p} / \mathrm{HIF}-1 \alpha /$ VEGF axis. Our study ascertained the therapeutic effects of circ-Gcap14 in an STZ-induced diabetic wound healing mouse model. 


\section{Acknowledgments}

This research was funded by Beijing Municipal Natural Science Foundation [Grant no. 7192160].

\section{Potential Conflict of Interest}

The authors have no conflicting financial interest.

\section{References}

1. Haddad JA, Haddad AN. The past decade in type 2 diabetes and future challenges. Hormones (Athens) 2018;17: 451-459

2. Davis FM, Kimball A, Boniakowski A, Gallagher K. Dysfunctional wound healing in diabetic foot ulcers: new crossroads. Curr Diab Rep 2018;18:2

3. Tao SC, Rui BY, Wang QY, Zhou D, Zhang Y, Guo SC. Extracellular vesicle-mimetic nanovesicles transport LncRNAH19 as competing endogenous RNA for the treatment of diabetic wounds. Drug Deliv 2018;25:241-255

4. Chalmers J, Joshi R, Patel A. Advances in reducing the burden of vascular disease in type 2 diabetes. Clin Exp Pharmacol Physiol 2008;35:434-437

5. Xu J, Liu X, Zhao F, Zhang Y, Wang Z. HIF1 $\alpha$ overexpression enhances diabetic wound closure in high glucose and low oxygen conditions by promoting adipose-derived stem cell paracrine function and survival. Stem Cell Res Ther 2020;11:148

6. Wang Z, Li H, Zhang D, Liu X, Zhao F, Pang X, Wang Q. Effect of advanced glycosylation end products on apoptosis in human adipose tissue-derived stem cells in vitro. Cell Biosci 2015;5:3

7. Hwang OK, Noh YW, Hong JT, Lee JW. Hypoxia pretreatment promotes chondrocyte differentiation of human adipose-derived stem cells via vascular endothelial growth factor. Tissue Eng Regen Med 2020;17:335-350

8. Wu H, Wu S, Zhu Y, Ye M, Shen J, Liu Y, Zhang Y, Bu S. Hsa circRNA 0054633 is highly expressed in gestational diabetes mellitus and closely related to glycosylation index. Clin Epigenetics 2019;11:22

9. Memczak S, Jens M, Elefsinioti A, Torti F, Krueger J, Rybak A, Maier L, Mackowiak SD, Gregersen LH, Munschauer M, Loewer A, Ziebold U, Landthaler M, Kocks C, le Noble F, Rajewsky N. Circular RNAs are a large class of animal RNAs with regulatory potency. Nature 2013;495:333-338

10. Hansen TB, Jensen TI, Clausen BH, Bramsen JB, Finsen B, Damgaard CK, Kjems J. Natural RNA circles function as efficient microRNA sponges. Nature 2013;495:384-388

11. Fischer JW, Leung AK. CircRNAs: a regulator of cellular stress. Crit Rev Biochem Mol Biol 2017;52:220-233

12. Geng W, Tang H, Luo S, Lv Y, Liang D, Kang X, Hong W. Exosomes from miRNA-126-modified ADSCs promotes functional recovery after stroke in rats by improving neurogenesis and suppressing microglia activation. Am J Transl Res 2019;11:780-792

13. Zhang AJ, Jiang T, Li Q, Jin PS, Tan Q. Experimental research on ADSCs-NCSS in wound repair. Exp Ther Med 2018;16:4429-4436

14. Qu Y, Zhang Q, Cai X, Li F, Ma Z, Xu M, Lu L. Exosomes derived from miR-181-5p-modified adipose-derived mesenchymal stem cells prevent liver fibrosis via autophagy activation. J Cell Mol Med 2017;21:2491-2502

15. Li Q, Xia S, Yin Y, Guo Y, Chen F, Jin P. miR-5591-5p regulates the effect of ADSCs in repairing diabetic wound via targeting AGEs/AGER/JNK signaling axis. Cell Death Dis 2018;9:566

16. Fernando EH, Gordon MH, Beck PL, MacNaughton WK. Inhibition of intestinal epithelial wound healing through protease-activated receptor- 2 activation in $\mathrm{Caco} 2$ cells. J Pharmacol Exp Ther 2018;367:382-392

17. Zhi Z, Yang W, Liu L, Jiang X, Pang L. Early missed abortion is associated with villous angiogenesis via the HIF-1 $\alpha$ / VEGF signaling pathway. Arch Gynecol Obstet 2018;298: 537-543

18. Lin CJ, Lan YM, Ou MQ, Ji LQ, Lin SD. Expression of miR-217 and HIF-1 $\alpha$ /VEGF pathway in patients with diabetic foot ulcer and its effect on angiogenesis of diabetic foot ulcer rats. J Endocrinol Invest 2019;42:1307-1317

19. Falanga V. Wound healing and its impairment in the diabetic foot. Lancet 2005;366:1736-1743

20. Zhang J, Chen C, Hu B, Niu X, Liu X, Zhang G, Zhang C, Li Q, Wang Y. Exosomes derived from human endothelial progenitor cells accelerate cutaneous wound healing by promoting angiogenesis through Erk1/2 signaling. Int $\mathrm{J}$ Biol Sci 2016;12:1472-1487

21. Li C, Wang Q, Shen S, Wei X, Li G. HIF-1 $\alpha$ /VEGF signaling-mediated epithelial-mesenchymal transition and angiogenesis is critically involved in anti-metastasis effect of luteolin in melanoma cells. Phytother Res 2019;33:798-807

22. Yu Z, Zhang T, Gong C, Sheng Y, Lu B, Zhou L, Ji L, Wang Z. Erianin inhibits high glucose-induced retinal angiogenesis via blocking ERK1/2-regulated HIF-1 $\alpha$-VEGF/ VEGFR2 signaling pathway. Sci Rep 2016;6:34306

23. Nan Y, Guo H, Guo L, Wang L, Ren B, Yu K, Huang Q, Zhong Y. MiRNA-451 inhibits glioma cell proliferation and invasion through the mTOR/HIF-1 $\alpha$ /VEGF signaling pathway by targeting CAB39. Hum Gene Ther Clin Dev 2018;29:156-166

24. Yu WY, Sun W, Yu DJ, Zhao TL, Wu LJ, Zhuang HR. Adipose-derived stem cells improve neovascularization in ischemic flaps in diabetic mellitus through HIF-1 $\alpha$ /VEGF pathway. Eur Rev Med Pharmacol Sci 2018;22:10-16

25. Wu J, Ke X, Wang W, Zhang H, Ma N, Fu W, Zhao M, Gao X, Hao X, Zhang Z. Aloe-emodin suppresses hypoxia-induced retinal angiogenesis via inhibition of HIF-1 $\alpha$ / VEGF pathway. Int J Biol Sci 2016;12:1363-1371 